\title{
Research on and Application of Digital Visual System of Power Communication's Indicators
}

\author{
Lijie $\mathrm{Wu}^{1, \mathrm{a}}$, Zhiyuan $\mathrm{An}^{1, \mathrm{~b}}$, Xinjian Shu ${ }^{1, \mathrm{c}}$,Yong Zhang ${ }^{1, \mathrm{~d}}$ \\ ${ }^{1}$ Information \& Telecommunication Co. of State Grid Henan Electric Power Company, \\ Zhengzhou 450052 China \\ a374663965@qq.com, ${ }^{\text {bxganzhiyuan@163.com, }{ }^{\text {c}} \text { sxji@chinaren.com, }{ }^{\text {d}} 8318389 @ q q . c o m}$
}

Keywords: Communication Indicator; DVS; Data Set; Physical architecture; Data architecture; Technical Architecture

\begin{abstract}
As the scale and the size of communication networks increase rapidly, power communication has stricter requirements in the communication scheduling intensification. This paper firstly analyzes the requirements of digital visual systems (DVS) of communication's indicators. It also classifies these indicators into several types, including scale type, operation type, surveillance type and check type. We build a big screen representation model of communication indicators. Then, the architecture of representation of communication indicators is illustrated mainly in physical architecture, data architecture and technique architecture. By implementing the visualization of communication indicators, we can effectively improve the efficiency of communication dispatch management and the intensification management level of communication domain.
\end{abstract}

\section{Introduction}

Visualization technology is the combination of scientific computing and graphics technology, it involves a wide range of disciplines and technologies, such as scientific and engineering computing, computer graphics, image processing, man-machine interface, and so on. Being a new technology, it has achieved a rapid improvement since its advent, and has been widely used in various disciplines ${ }^{[1]}$.With the scheduling intensive development of electric power information and communication, the requirements of communication scheduling for the network real-time monitoring, analysis and control are increasing year by year. The demand that graphics should be displayed intuitively is required. A variety of key indicators of information communication needs to be displayed in the screen completely, accurately, in real-time and in a batch mode. By displaying function and background data of the longitudinal transverse real-time interaction, the system is required to have a warning effect on the running status of information and communication, and to provide the accurate auxiliary decision-making for information communication scheduling dispatcher, the automation and monitoring automation of information and communication systems running, service routing, display interaction. Therefore, it can benefit the power information communication run intensively, standardization, intelligent management.

\section{Requirement of the Communication Indicators Display}

In the Visual display of DVS (Digital Visual System) System, the power communication scale, operation, monitoring and assessment indicators are expressed on the screen clearly ${ }^{[2]}$.It makes the data more clear and intuitive. Thus, the working efficiency of the communication dispatcher can be improved greatly.

(1) Communication network monitoring

DVS treats the backbone transmission topology as the main framework, monitors and controls communications equipment running status in real-time. It mainly monitor communication transmission network topology, network elements, alarm status, switching network and data network system operation. 
(2) Equipment and network scale

By using histograms and line charts to DVS shows the station quantity, the cable length, the number of the optical transmission equipment, the communication power equipment quantity, the communications switching equipment quantity, the coverage of substation fibers, the optical fiber communication network coverage at the grass-roots level substation equipment and network scale and growth of index data. In this way it shows the current state of the site size and fiber size.

(3) Communication operation class

This type of indicators mainly consists of communication maintenance indicators, operation mode indicators and defect management operation indicators and so on, such as communication maintenance plan completion rate, the number of complete project, communication unplanned maintenance rate, number of project, monthly methods single quantity, methods single archiving rate, monthly defect single number, and defect rate of single archive, etc. ${ }^{[3-5]}$.

(4) Assessment indexes

The circulation efficiency of the control the repair order of indicator assessment consists of transmission light path production rate and maintenance schedule file, defect rate, running record filing rate, rate of archive, the alarm confirm rate, visit rate, channel correlation rate, the ways of business and the rate of single link. Communication indexes of visual display requirements are shown in figure 1.

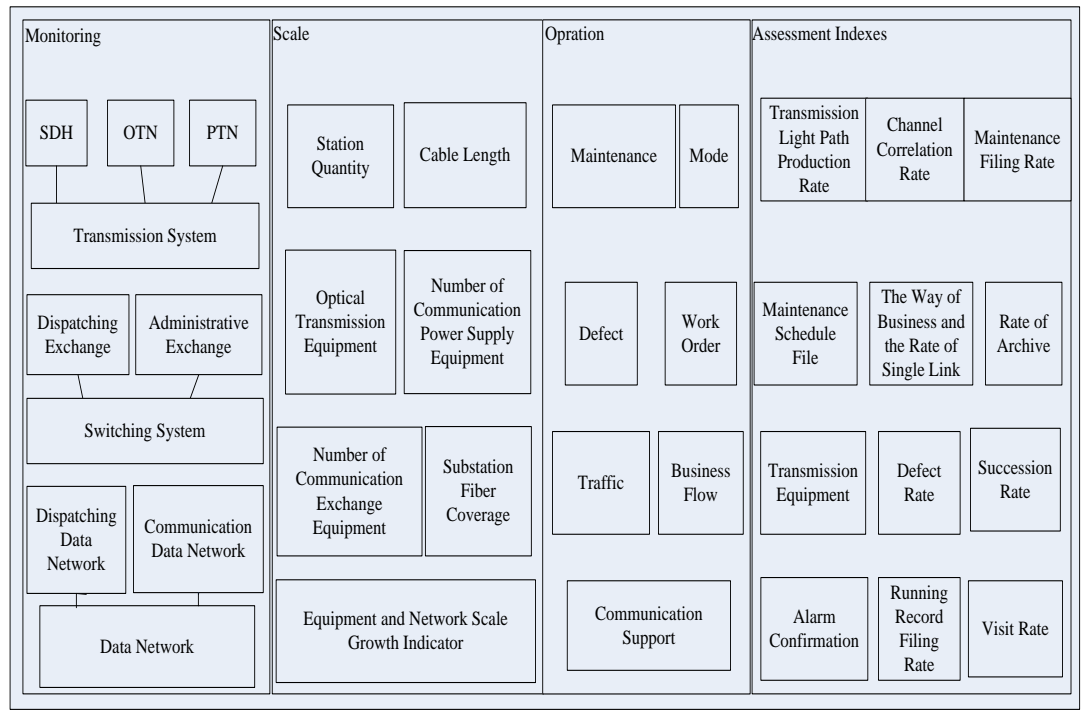

Fig.1 the diagram of communication index of display requirement

\section{Visualization Construction Principle}

Visualization technology can be used as analysis tool. By show a large number of things graphically, it transforms the abstract data into intuitive information and makes these data easy to understand, thus, it can help the analysts find out the problem on the huge amounts of data ${ }^{[6]}$. Its application should strictly follow the following principles:

(1) Practical principles

It application should give priority to the practical need, considers both advanced and forwardlooking properties, to satisfy the demand of real-time online monitoring work.

(2) Reliability principles

Using redundant configuration system is the key link in the hardware and software resources, thus, it can ensure safe and reliable operation of the system.

(3) Scalability principles

It should use the flexible design to improve the system scalability and realize the adjustment of business and system synchronization.

(4) Economy principles 
It should make full use of existing information monitoring center screen hardware and the operation of the information communication system to save the cost of investment.

(5) Provide data support based on the existing system

It should exploit resource data communication management system and build standardized data platform to complete communication indicators shown in the monitoring center.

\section{Building the Indicator Representation Model}

Establishing the perfection of the mechanism of power communication professional indicators and data analysis requires fundament on the analysis of model tools ${ }^{[7]}$, comprehensive diagnostic analysis power telecommunication network scale, network operation, monitoring and so on.The index data visualization display of communication model has three layers: data layer, index layer and application layer. The diagram of index data visualization display model is shown in the figure 2.

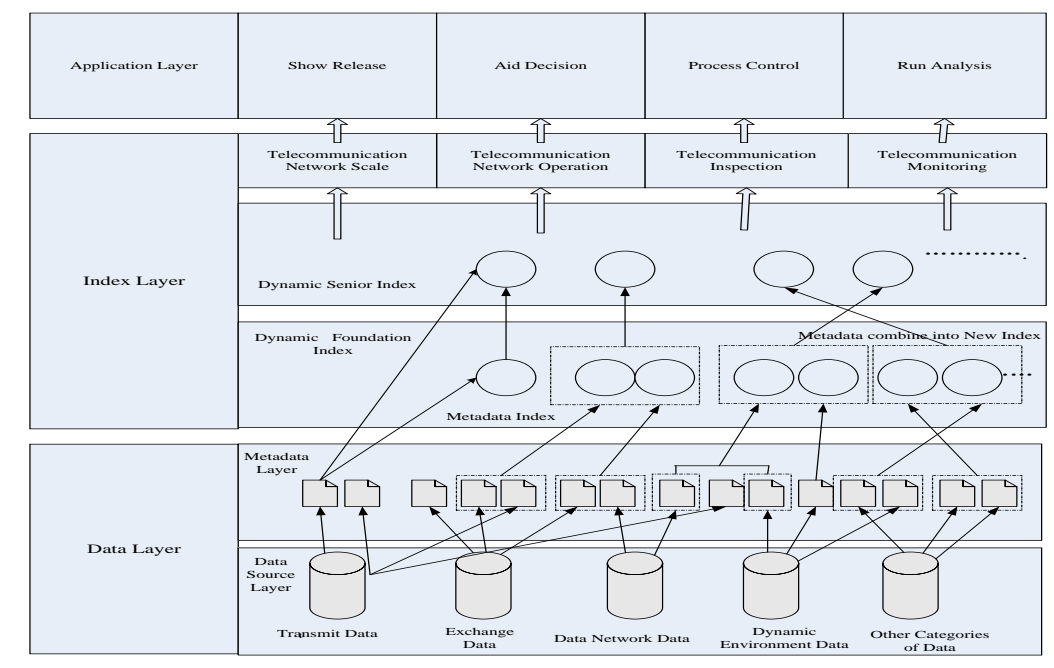

Fig.2 model of index data visualization display

(1) Data layer

By setting up power communication data resources platform into different levels, the automatic data acquisition can be realized, obtained and released. First, the data source layer collects the professional network management data through the north interface, and then sends to the metadata layer. In the metadata layer, metadata is extracted dynamically to remain the only source.

(2) Index layer

Index layer specifies index definition, sources, acquisition methods, statistical calibre, publishing and sharing. This layer is divided into dynamic foundation index layer and dynamic senior index layer. Dynamic foundation index layer will first collect the data layer metadata, and then combine the metadata into a new index, finally send the new index to dynamic high index layer. These new indexes are classified separately to four categories: telecommunication network scale, telecommunication network operation, inspection index and monitoring index.

(3) Application layer

The data of communication operation indexes is used to set up all kinds of auxiliary decision analysis model. Based on the data correlation analysis, the data of communication assessment index is used to build the whole communication process in each link to control the operation of system, and to coordinate communication at the level of management and operation work. Cutting communication sharing data in two levels of assessment index, and storage the linkage of the network data, rack optimizing communication departments at different levels are arranged for. Operation mode, operation analysis, process control and fault disposal of all the running work provides online analysis and auxiliary decision support. Finally, these indicators are displayed on the screen in various graphical forms. 


\section{Overall Architecture Design}

All kinds of technologies are needed to support the visual display the communication indexes. The visual system is mainly composed of three parts: physical architecture, data architecture and technical architecture.

\section{1 physical architecture}

Visual display system is divided into two parts, including software and hardware. In order to make full use of existing resources, power communication indicators visual show platform can be built on the basis of existing information. It can take advantage of the enterprise information bus which is based on service-oriented architecture. The architecture gives data a series of extraction, transformation, finishing, and forms a uniform data information sharing layer. Software system consists of three layers of C/S software architecture. It saves images and data in the database, which is read by the application server, pushes the picture and data to the graphic workstations. Then pictures will be broadcasted by the graphic workstation player.

Hardware system through the graphic workstation outputs the picture signal to the stitching controller, where the signal is put on the big screen to display. The display of the graphic platform, uses object-oriented design method. It can be defined for describing and using different components of class. It can also put the various electrical components as the smallest unit of graphics. The graphics for layered implementation is shown in figure 3.

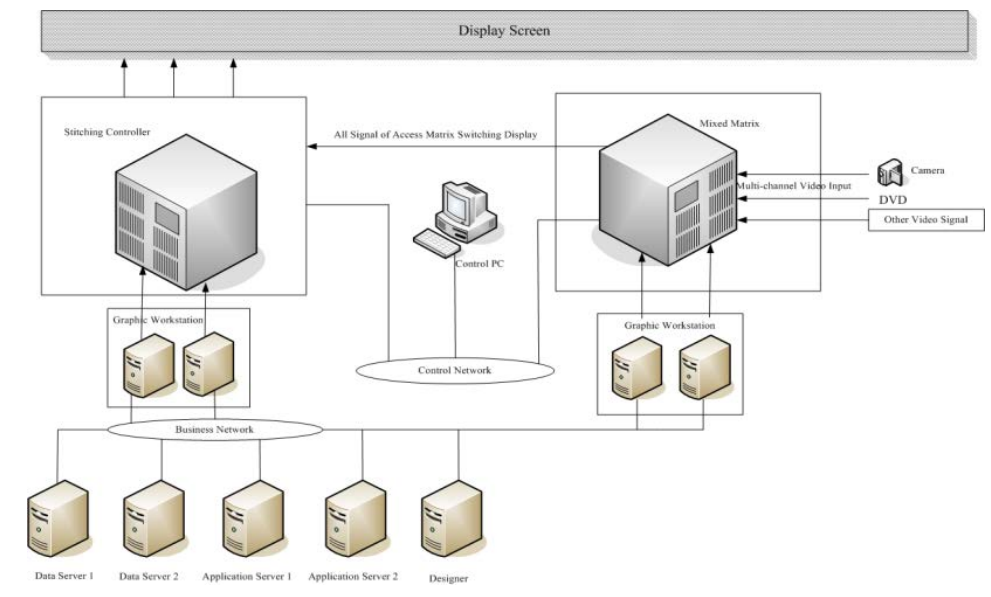

Fig.3 the physical architecture diagram of visualization system

\section{2 data architecture}

A lot of data can be collected from various information and communication systems. These data becomes information after being interpreted and given a certain meaning. Data processing includes the data collection, storage, retrieval, processing, transformation and transmission. The basic purpose of data processing is to extract and to derive useful information for us from a large number of data which maybe messy and difficult to understand. 


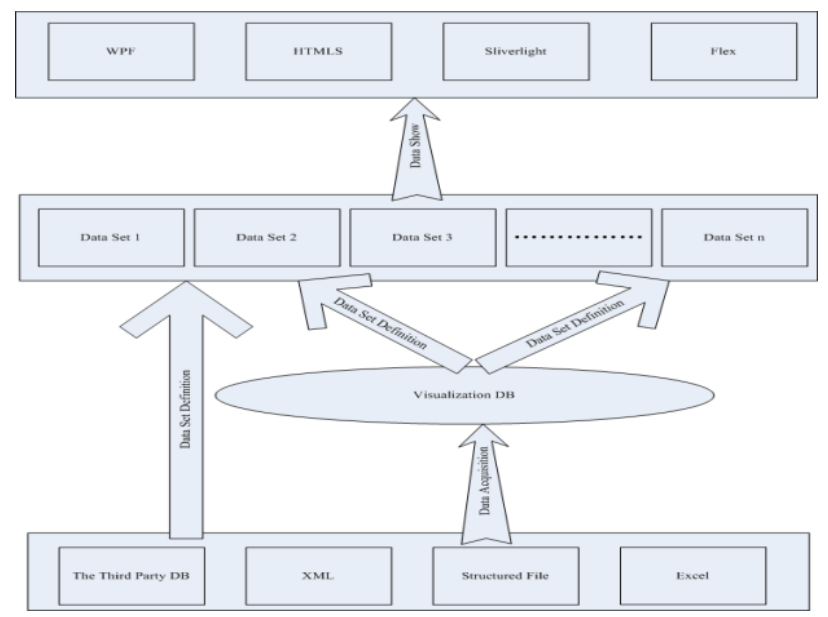

Fig.4 the data structure of screen visualization system

Data processing of the Screen visualization display platform is mainly divided into data acquisition, data processing and data show. The metadata model mainly includes data acquisition, collection service definition, data display, index model, dimensional model. Its data structure is shown in figure 4.

After data collection, data processing is necessary. Data processing is mainly responsible for the visual data statistics, analysis, calibration, cleaning and so on. It will eventually output data to display data set for service invocation.

\subsection{Technical architecture}

Visualization platform contains two parts: server part and show part. After extracting data from various application systems, file and database, a deep processing is done via JMS, SOAP and FTP protocol, and finally is designed and displayed by show part.

The diagram of technical architecture is shown in figure 5.

Service contains visual service framework, data acquisition, data processing, data push, visualize, data management, data storage, historical data management, etc.

Visualization services framework mainly includes timing schedule, interface release, core packages, and integrated data platform. These business components are used to support the data collection, processing, delivery, storage, and other functions. It is the core of visualization services. After acquisition and processing, data can be extracted to the server part for further processing.

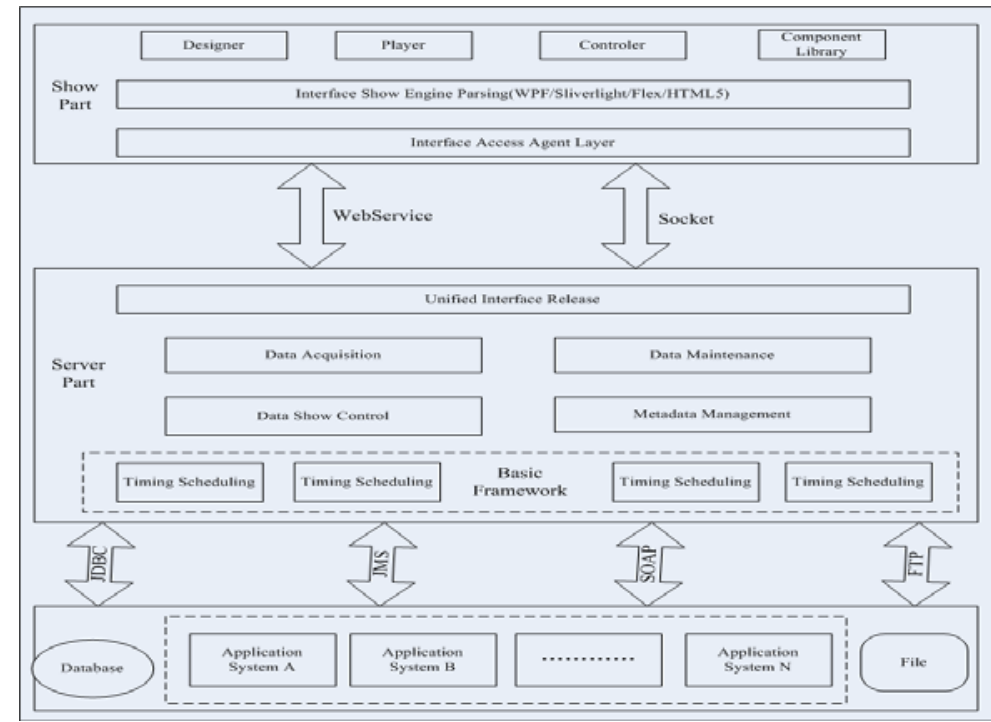

Fig.5 the diagram of visualization technical architecture 
The show part includes show engine parsing, adaptation, interactive control, showing picture, visual designer and visual controller.

(1)Visual display parsing engine: containing display description file format definition, development description file parsing engine, etc.

(2)Visual designer: containing interface framework, editing, graphic picture preview, data binding, index maintenance, style, version management, property settings, etc.

(3)Visual display terminal: displaying the parsing engine output to in the screen. It contains interactive control module, develop adaptation screen, desktop, mobile and touch-screen display adapter.

(4)Visual controller: requiring to develop the control end similar to PowerPoint remark presentation mode. There is also interactive control function in it.

\section{Conclusions}

With the increases of the network scale and number, more advanced and more intuitive tools and means are needed to help the communication dispatcher to achieve the timely and effective monitoring communication, communication metrics visualization display design. A research on and application of DVS of power communication's indicators is analyzed in this paper. The introduced DVS can provide technical support for information communication scheduling intensive management, operation of auxiliary decision-making for dispatcher accident analysis, risk prevention, early warning and a powerful platform for the display. After communication indicators visual display, the proposed system can greatly improve the working efficiency of the power grid companies, improve the quality of service, and then achieve the demand of the modern intelligent power grid.

\section{References}

[1]Zhong Deng-hua, Water resources and hydropower engineering construction site layout visualization research dynamic demonstration system [J]. Journal of water resources and hydropower technology, 2001, 12 (32):10-15.

[2] Chen Xiao-lian. Visualization of electric power communication network resource management [J]. Power system communications, 2005, 26 (156):26 to 30.

[3]JOverbye Power System Visualization [J].Power system automation,2005,29(16):60-65.

[4] Zhao Rui. Power system visualization technology and its application in Beijing power grid [D]. Beijing: north China electric power university, 2012.

[5]Li Qian. Power system visualization technology and its application in smart grid dispatching [D].Shandong, Shandong University, 2009.

[6] Li Da-yong and so on. Grid information visualization application study [J]. Power system protection and control, 2009, 23 (37):156-158.

[7]Li Rui-qing. Power grid enterprise information visualization of the construction of the exploratory study [J]. Journal of east China power, 2009, 5 (2):205-209. 